\title{
Multilayered injection of calcium hydroxylapatite filler on ischial soft tissue to rejuvenate the previous phase of chronic sitting pressure sore
}

\author{
This article was published in the following Dove Press journal \\ Clinical, Cosmetic and Investigational Dermatology
}

\section{JongSeo Kim}

Kim-Jongseo Plastic Surgery Clinic, Seoul, Republic of Korea

\section{Video abstract}

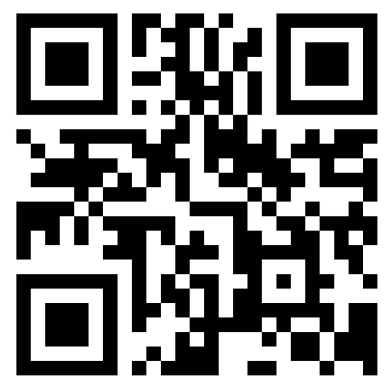

Point your Smart Phone at the code above. If you have a QR code reader the video abstract will appear. Or use: https://youtu.be//FPEBJIMmrM
Correspondence: JongSeo Kim Kim-JongSeo Plastic Surgery Clinic, 2F ApGuJung bd, 842-NonHyun-ro, GangNam-gu, Seoul 06025, Republic of Korea

Tel +82 10-9026-0000

Email plastic@surgery.co.kr
Introduction: During a sitting position, the pressure distribution is located below the ischial tuberosity. Many women have skin atrophy on the ischial area. To treat atrophic changes on the skin above the ischium, volumization and improving skin texture are acquired simultaneously. Two methods of automatic and manual injections using a hard filler with a stitmulating effect were administered respectively to both the dermis and subdermis layers. A biopsy study using various straining evaluated histological tissue reactions after the filler injections.

Methods: This study focused on rejuvenating soft tissue on the atrophic ischeal areas, as described by the author as the previous phase of chronic sitting pressure sore, by using the multi-layered injection of calcium-hydroxylapatite (CaHA) filler. Sixteen women (mean, 38.5 years) were treated from 2012 January to 2019 April. Prior to the injection, $1.5 \mathrm{cc}$ of Radiesse ${ }^{\mathbb{R}}$ (calcium hydroxylapatite filler; Merz, Germany) was diluted with $1 \mathrm{cc}$ of normal saline and $0.5 \mathrm{cc}$ of lidocaine, and $3 \mathrm{cc}$ of filler mixture (1:1 dilution) was made. All subjects received the intradermal injection and multi layered subdermal injection with $2.5 \mathrm{cc}$ of diluted CaHA filler. A second session for booster treatment was performed at 6 months using the same method. Photography was taken by a camera and a dermascope observation before and 7 months after. Before and 7 months after the first injection, soft tissue depression, skin discoloration, and roughness were assessed. Standard deviations and coefficients of variation were also calculated for changes in depression, discoloration and roughness after the treatment. Biopsy specimens $(3 \times 5 \mathrm{~mm})$ were taken from three patients 7 months after the first session. The specimens were analyzed using various stainins.

Results: The improvements of skin quality, skin fold, and roughness were visible at physical examination, medical photography and also at high-resolution dermascope examination in all patients. Post-treatment the depressed amounts on the ischial areas reduced with increased volume. Conclusion: Depressed soft tissue and skin folds on ischial areas were significantly improved by volumization of subdermal filler injection. The skin quality, roughness, and pigmentation on ischial areas improved, and these improvements may be caused by intradermal micro-droplet injections of CaHA filler which may be influenced by neocollagenesis by numerous fibroblasts and increased micro-blood circulation (neovascularization). This is the first article to show the scientific evidence of neocollagenesis and tissue reaction after an injection of CaHA filler in the dermis, especially using various histological staining and to show various stages of inflammation and foreign body reaction around $\mathrm{CaHA}$ particles. Numerous fibroblasts were present around CaHA particles, but plasma cells were not found. Interestingly a few eosinophils were found around CaHA filler. After a significant period of time, multi-layered injections of diluted CaHA tightened and remodeled atrophic ischial skin. The multi layered injection approach was safe and effectively treated ischial soft tissue atrophy without significant side effects, such as infection or delayed swelling or lumps.

Keywords: calcium hydroxylapatite filler, ischial pressure sore, skin roughness, neocollagenesis, tissue reactions 


\section{Introduction}

Women were not allowed to show the specific skin areas of the body up until recently. Whereas in the new generation's society, some women want to steal the spotlight in skimpier swimming wear or string pants and show off their glamorous figure. The string pants style is a new trend that shows too much buttocks. In today's society, women are working, reading, and studying in the sitting position. Except for the time to lie down and stand up, always women are sitting in a chair. However, the sitting position for a long time puts pressure on one's ischial area. Therefore, buttocks are sore from sitting too long which decreses blood circulation. Sustained pressure can cut off circulation to vulnerable parts of the body, and soft tissues including fat and dermis can atrophy. Prolonged injured tissues on the ischial areas can develop thinning of the dermis and fatty layers, and leading to soft tissue atrophy including soft tissue depression, skin discoloration, and roughness. Treatment thus requires combining subdermal volumization with skin texture improvement including collagen stimulation in the skin.

Because buttock skin is a thick and weight-bearing area, harder fillers are preferred to lift the depressed area than the usual hyaluronic acid (HA) filler. Also, calcium hydroxylapatite (CaHA) filler induces neovascularization and neocollagenesis, ${ }^{1,2}$ and these effects can help to treat pigmentation and skin roughness on the ischial area. CaHA filler is approved for the aesthetic treatment of deep facial rhytids and hand dorsum rejuvenation. ${ }^{3,4}$ As the most abundant structural protein in the dermal extracellular matrix (ECM), collagen type $I$ is fundamental for skin strength and resilience, ${ }^{5}$ and its production is stimulated by CaHA. ${ }^{6}$

To improve depression deformities and skin folds on the ischial area, subdermal injections may be an effective treatment. While only the subdermal injections dermal filler was ineffective at changing or improving skin texture, it did have pronounced subdermal volumizing effects (ballooning effect). ${ }^{7}$ Therefore to improve atrophic skin conditions and texture such as discoloration and fine wrinkles, the intradermal injection may be a proper treatment. In this study, CaHA filler was injected into multi layers (dermis, subdermis using dermal scraping technique, and fat layer) on the ischial area.

"Previous phase of chronic sitting pressure sore on buttock areas" was categorized and measured. Skin roughness was evaluated by areal topography with optical technique and microphotography, which is the ordinary way to obtain the image skin texture and the degree of skin irregularity. ${ }^{8,9}$ Surface mapping also was done by simple trigonometric calculations. The degree of the skin discoloration or pigmentation on the ischial area was assessed by a Wood's lamp. To evaluate tissue reaction such as neocollagenesis after the injection, biopsies were taken and stained using various methods.

\section{Materials and methods}

\section{Patients}

This study is a prospective, open-label study for soft tissue rejuvenate of the previous phase of chronic sitting pressure sore using the multi-layered injection of CaHA filler on ischial areas. Sixteen women, 27-49 years old (mean, 38.5 years), were treated from 2012 January to 2019 April. Subjects had conspicuous ischial depressions and dark discolorations. Study protocols and consents were approved by the Korean Plastic Surgery Society. The study protocol conformed to the guidelines of the 1975 Declaration of Helsinki. An explanation of risks, benefits, and potential complications was given to all subjects and written informed consent was obtained before treatment.

\section{Injection methods}

Before injection, $1.5 \mathrm{cc}$ of CaHA filler (Radiesse ${ }^{\circledR}$, Merz, Germany) was diluted with $1 \mathrm{cc}$ of normal saline and $0.5 \mathrm{cc}$ of lidocaine, and $3 \mathrm{cc}$ of filler mixture (1:1 dilution) was made. All subjects received the intradermal injection and multi layered subdermal injection with $2.5 \mathrm{cc}$ of diluted $\mathrm{CaHA}$ filler. For both sides, $2 \mathrm{cc}$ of the filler mixture was injected into subdermis into both ischial sides with a $27-\mathrm{G}$ cannula in 200 passes. At first, 50 strokes were injected just under the dermis using skin scraping technique for skin stimulation, and the remaining 150 strokes were injected into deep fat layers to create a volumizing effect. The injection amount of CaHA per pass was 0.01cc and actual CaHA was $0.005 \mathrm{cc}$. And then $0.5 \mathrm{cc}$ of filler mixture was injected into the dermis. To inject exactly into dermis evenly with less effort, XeoBel injector ${ }^{\circledR}$ (ContacKorea, South Korea) was used. The injector had 9 needles $(31 \mathrm{G})$ in needle head part, and one pass made 9 injection sites. With the injector $0.5 \mathrm{cc}$ of diluted CaHA was injected dividing into $0.001 \mathrm{cc}$ (microdroplets) through 500 injection sites for both sides. Among the $3 \mathrm{cc}$ diluted filler mixture, $0.5 \mathrm{cc}$ of diluted CaHA was not used. A second session for booster treatment was performed at 6 months using the same method. 


\section{Outcomes assessment}

Photography was taken by a camera (EOS Kiss Digital X, Cannon, Japan) and a dermascope (COSCAM CCL215USB, Sometech, South Korea) observation before and 7 months after. Before and 7 months after first injection (1 month after second booster injection), soft tissue depression (5 scales using a special ruler), skin discoloration (using Wood's lamp), and roughness (trigonometric calculations) were assessed. Standard deviations and coefficients of variation were also calculated for changes of depression, discoloration, and roughness after the treatment.

\section{Depression}

Depressed ischial areas were measured using a cannula ruler through a pin hole on a transparent and circular acryl plate $(5 \mathrm{~cm}$ diameter) before and after 7 months after treatment. In a prone position the acryl plate was placed on depressed ischial areas just with the plate's own weight. The guide cannula was then inserted through the pin hole of the acryl plate (without touching the acryl plate) to measure the depressed amount of the ischial area. Depressed ischial areas were categorized as follows:

4 - severe depression more than $2 \mathrm{~mm}$;

3 - moderate depression more than $1 \mathrm{~mm}$;

2 - mild depression less than $1 \mathrm{~mm}$;

1 - flat surface, no depression (almost all area of the acryl plate are in contact with the ischial area with its own weight of the acryl plate);

0 - convex curvature (only central area of acyl plate is in contact with the ischial area).

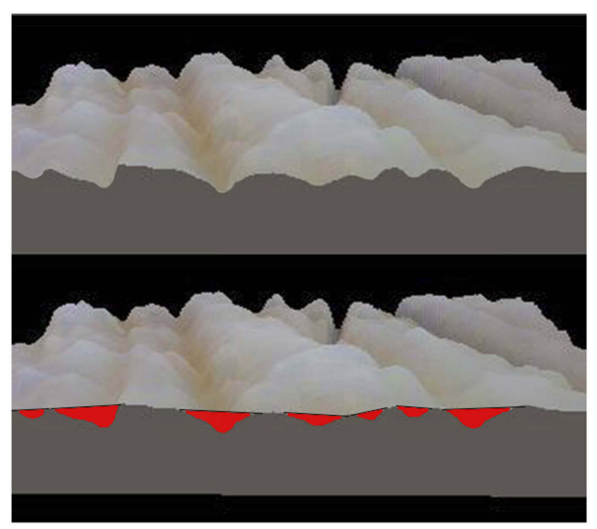

\section{Skin roughness using microscopic trigonometric calculations}

Photos were taken using a dermascope. Skin roughness was then evaluated by areal topography with optical technique. ${ }^{8}$ Surface mapping was done, ${ }^{10}$ and the patchwork method determined the sum of triangle areas $(1 / 2 \times$ base $\times$ height of a triangle $) .{ }^{11-13}$ In the smooth skin surface with a low degree of roughness, the sum of the triangular areas was small and showed a nearly horizontal line. The larger areas of triangular patches represent a more severe degree of skin roughness (Figure 1).

\section{Skin discoloration}

The degree of the skin discoloration or pigmentation on the ischial area was also assessed by a Wood's lamp, which is a device that emits ultraviolet light within the $365-\mathrm{nm}$ range and is commonly used by dermatologists to assist in the diagnosis of various pigment and infectious disorders. The examination was performed in a dark room, allowing the Wood's light to shine directly on the affected area for 5 seconds and to look for dark discoloration or any changes in color or fluorescence. Normal skin does not fluoresce under the light of a Wood's lamp, so only discoloration was measured. In addition, subtle changes in color were examined. The degrees of dark discoloration of the ischial areas were categorized as follows:
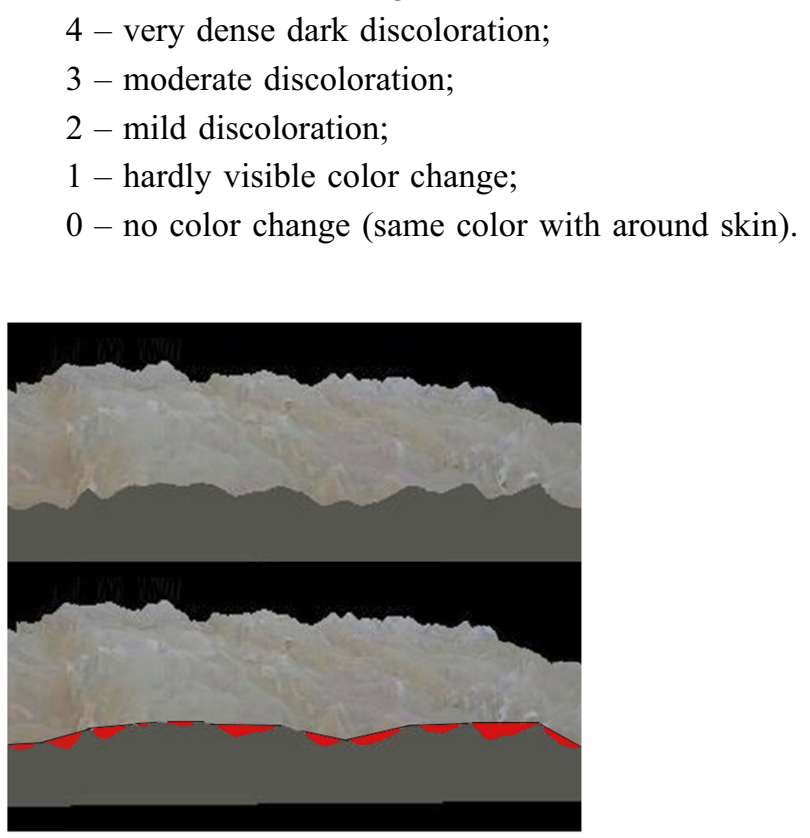

Figure I Skin roughness was evaluated by the areal topography with an optical technique using a microscope. Surface mapping was made, and the patchwork method was determined by the sum of triangle areas ( $1 / 2 \times$ base $\times$ height of a triangle). Before treatment, the sum of the triangle areas was 366 (left panel). Seven months after treatment, the sum of triangle areas decreased to 231.5 , and it revealed that roughness of skin improved in the ischial area after multi layered calcium hydroxylapatite (CaHA) injection (right panel). 


\section{Histologic study for neocollagenesis}

Biopsy specimens $(3 \times 5 \mathrm{~mm})$ were taken from three patients 7 months after the first session (1 month after the second booster session). Inflammation and foreign body reaction are the body's response to filler injection. Trauma by needle or cannula also can cause direct damage to cells in the immediate area of injury which may include bleeding. Filler injection, mechanical trauma, and minor bleeding initiated a cascade of events in the inflammatory processes that promoted healing of the soft tissue. There are some consequential processes such as wound healing, including the three stages of inflammatory, proliferative and maturation, and foreign body reaction. An acute vascular inflammatory response cannot be observed at that biopsy time, but repair-regeneration stages of wound healing and remodeling and maturation stage were observed. Therefore, two different stages of wound healing around CaHA particles 1-month and 7 months after injection were observed, such as repair-regeneration stages. Early to late foreign body reactions were also observed around 1-month particles and 7-month particles. Tissue reactions with various inflammation cells and morphology of CaHA filler particle were analyzed by various staining methods such as H\&E stain, PicroSirius Red (PSR) stain, Herovici's stain, Masson's Trichome stain, and immunohistochemistry (IHC) of type I and III Collagen.

\section{Morphologic study for particle shape and size of CaHA using SEM}

Remaining (not be injected) diluted CaHA filler was scanned with a CX-200TA scanning electron microscope (SEM: COXEM, South Korea) to determine exact particle sizes and shapes. Using SEM, photographs at a magnification of $15 \times$ to $300,000 \times$ and a maximum resolution of 3.0 $\mathrm{nm}$, under a $20 \mathrm{KeV}$ voltage and "SE" scanning mode. In this study the images were captured at $300 \times 500 \times$, $1,000 \times, 3,000 \times$, and $5,000 \times$ to see particles of CaHA.

\section{Results}

The skin quality, skin fold, and roughness improvements were visible at usual photography and also at highresolution dermascope examination in all patients (Figures 2-4). The depression amounts on ischial were reduced and volumized after the treatment (Figures 2 and 3).

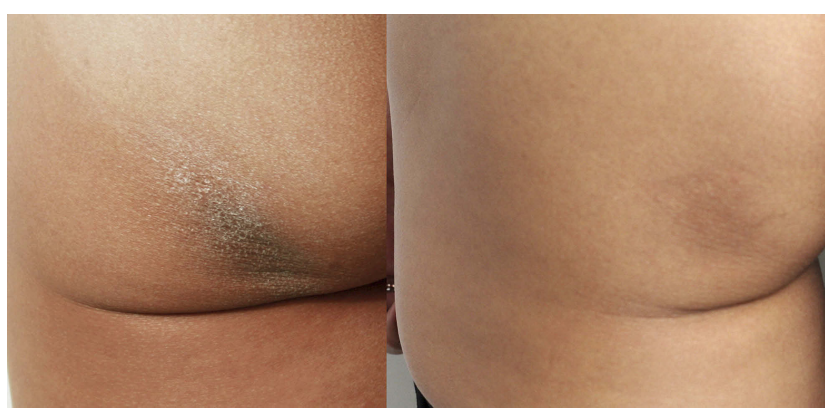

Figure 2 Photographs with the same lighting for a 39-year-old women who had previous phase of chronic sitting pressure sore. Before treatment (left panel). After 7 months post injections of diluted $\mathrm{CaHA}$, depressed ischial areas were volumized, and dark pigmented skin was also treated (right panel).

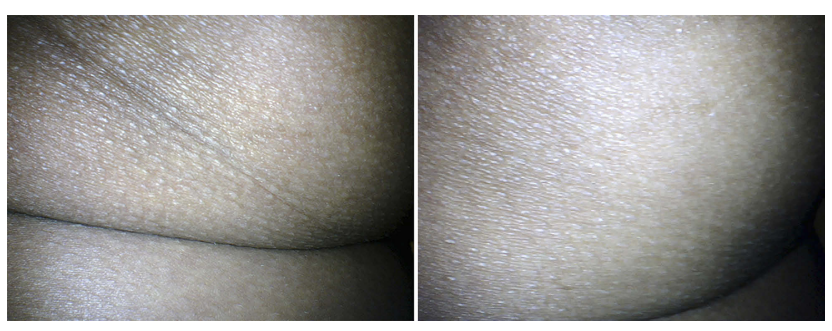

Figure 3 Low magnification of dermascope view (10x). Before treatment, there were oblique skin folds on buttock with scale-4 depression (more than $2 \mathrm{~mm}$ ) (left panel). Seven months after treatment, oblique skin folds, and the depression were improved (right panel).
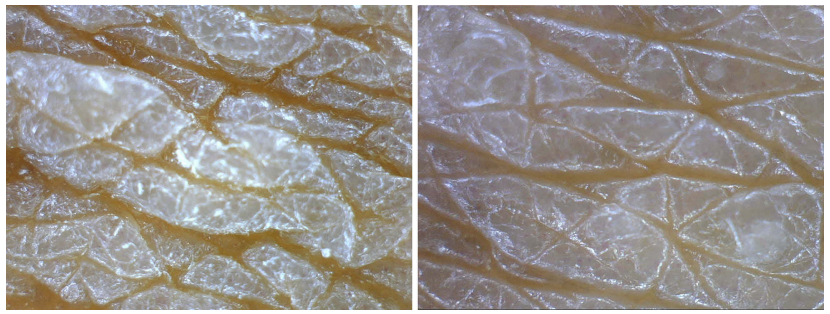

Figure 4 High resolution of dermascope view (100x). Before treatment, the skin was rough and chapped with keratinization (left panel). Skin roughness improved and looked smooth with less keratinization on the surface (right panel).

\section{Ischial depressions}

The depressions improved $1.50 \pm 0.70$ scales from 3.03 \pm 1.41 to $1.53 \pm 2.12(p<0.001)$ (Table 1). All ischial areas showed improvement of depression deformity.

\section{Skin roughness}

The skin roughness significantly improved in photography (Figure 2) and microscopic observations (Figures 3 and 4). The skin roughness also statically improved in "microscopic trigonometric calculations" (Figure 1). The microscopic trigonometric calculations (skin roughness index) improved $18.60 \pm 4.65 \%$ from $326.56 \pm 42.07$ to 265.34 
Table I Improvement of depressed areas, skin roughness, and skin discoloration

\begin{tabular}{|l|l|l|l|}
\hline & Depression & Roughness & Discoloration \\
\hline Before & $3.03 \pm 1.4 \mathrm{I}$ & $326.56 \pm 42.07$ & $2.59 \pm 0.7$ \\
After & $1.53 \pm 2.12$ & $265.34 \pm 45.96$ & $1.96 \pm 0.7$ \\
Improvement & $1.5 \pm 0.70$ scales & $18.6 \pm 4.65 \%$ & 0.62 scales \\
\hline
\end{tabular}

Note: After two sessions, 7 months post injection, depression improved 1.50 scales from 3.03 to 1.53 ( $p<0.00 \mathrm{I}$ ), skin roughness improved $18.6 \%$ from 326.56 to $265.34(p<0.001)$. discoloration improved 0.62 scales from 2.59 to $1.96(p<0.05)$.

$\pm 45.96(p<0.001)$ (Table 1). All ischial areas showed improvement of skin roughness.

\section{Skin discoloration}

The skin discoloration improved 0.62 scales from $2.59 \pm 0.7$ to $1.96 \pm 0.7$. $(p<0.05)$ (Table 1$)$, and lighter and pinker. Among 32 ischial areas of 16 patients, 14 ischial areas showed no improvement of discoloration (Figure 2).

\section{Histologic finding after CaHA filler in dermis}

H\&E (Figures 5-10), Herovici (Figure 11), Trichrome staining (Figure 12), IHC-Collagen-I, IHC-Collagen-III (Figure 13), and PSR (Figures 14 and 15) slides were photographed within exactly the same dermis areas. By all accounts in the same areas of the dermis, new collagen-

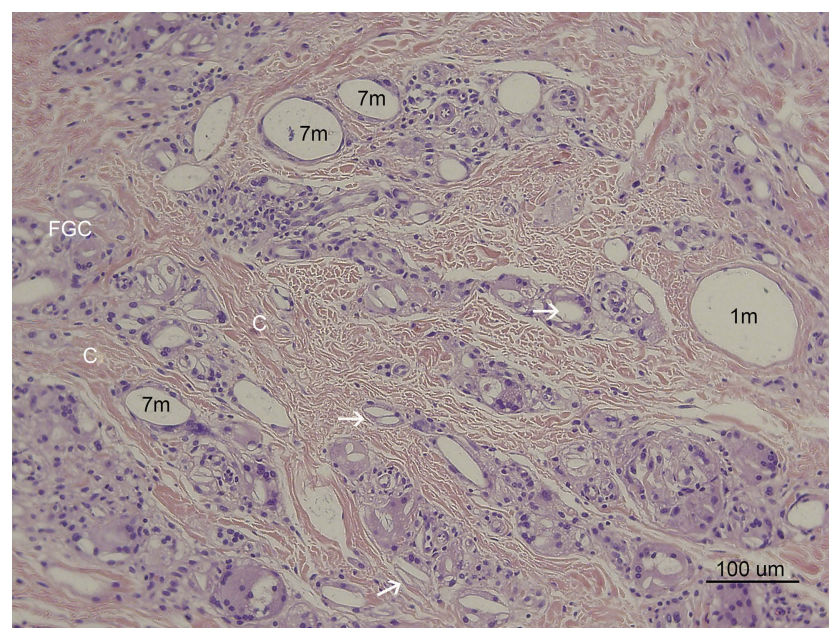

Figure 5 H\&E stain (I00x magnification) showed inflammation cells (FGCs, lymphocytes, macrophages) and collagen deposition in the dermis of treated ischial area. There were various sizes of CaHA particles. Larger than 40-micron particles might be injected I month before the biopsy (I m: I month) and a thin collagen layer was observed around the particle $(1 \mathrm{~m})$ without infiltration of the giant cell. There were many fibroblasts around the particles $(1 \mathrm{~m})$ (see Figure 6). Whereas dense deposits of thicker coarse-shape reinforced collagen fibers (C) were observed around smaller than 20-micron particles $(7 \mathrm{~m})$ that were injected 7 months before the biopsy. Around smaller than 20-micron particles $(7 \mathrm{~m})$, numerous FGCs and macrophages were also observed. FGCs ingested and cleaved CaHA particles (white arrows) into smaller sizes during chronic inflammation.

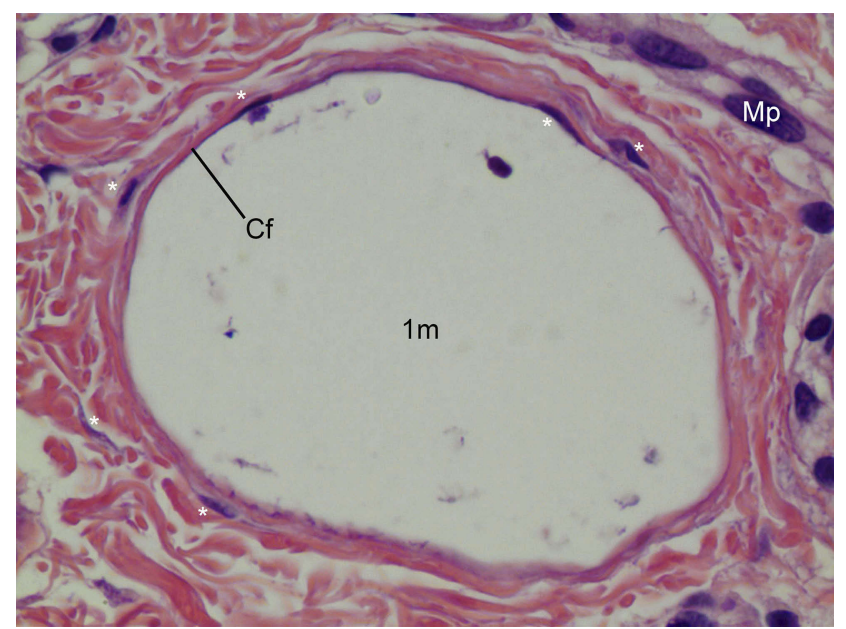

Figure $6 \mathrm{CaHA}$ particle $(\mathrm{I} \mathrm{m})$ in Figure 5 with 400x magnification. The large size particle was likely injected recently and particle might be injected I month before the biopsy. The particle was not surrounded by giant cells or macrophages (Mp), only fibroblasts $\left({ }^{*}\right)$ as shown here. This CaHA particle $(\mathrm{I} \mathrm{m})$ was surrounded by five fibroblasts, which may have contributed newly made thin collagen fibers (Cf), even at I month post injection.

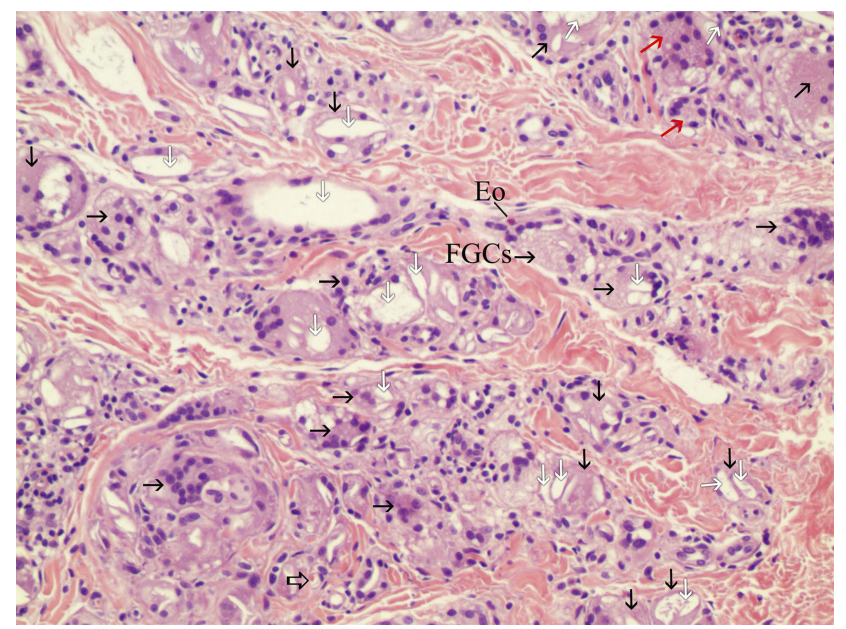

Figure $7200 \times$ magnification on the left area of Figure 5 (H\&E stain). Particles smaller than 20 microns were assumed to be particles that were injected 7 months before the biopsy and demonstrated late stage of inflammation in tissue reaction. FGCs (black arrow) ingested and cleaved CaHA particles and showed $\mathrm{CaHA}$ biodegradation. FGCs always positioned between new collagen fibers and CaHA particles (white arrows). The nuclei were centrally placed and overlapped each other in most FGCs. In some FGCs, ingested CaHA particles had displaced nuclei of FGCs peripherally. But in a few giant cells, the nuclei were arranged on the border as horseshoe shape like Langhans giant cell (red arrow). New vessels (empty black arrows) with a red blood cell its inside was visible and and demonstrated neovascularization by $\mathrm{CaHA}$ filler. A eosinophil (Eo) was found.

I\&III were created around particles of CaHA filler materials. Various sized CaHA particles were detected in the dermis (Figures 5-15). Some of CaHA-particles (CaHAPs) were bigger than 40 microns in size with round shape, and other particles were smaller than 20 microns in size with irregular shape in the same slides. "Bigger than 40 


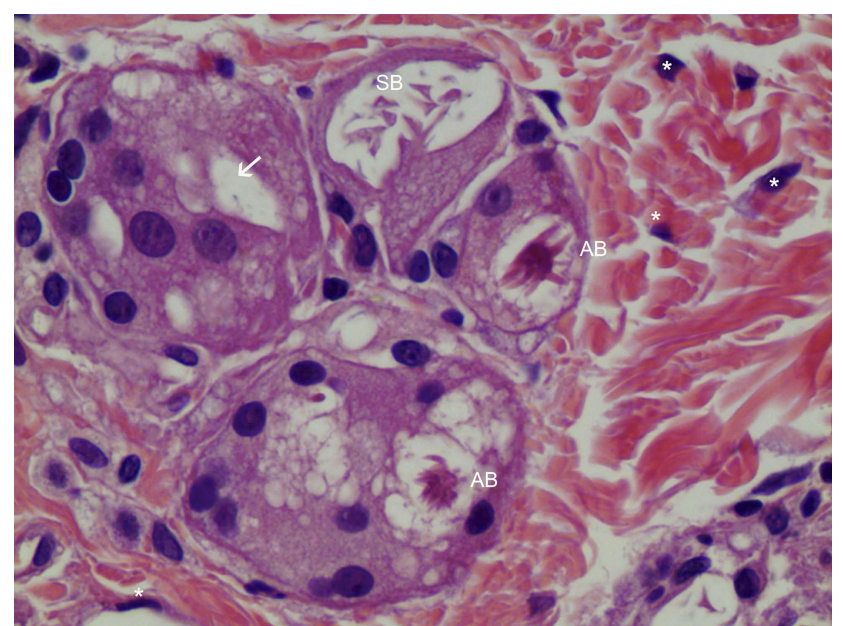

Figure 8 FGCs with asteroid body in H\&E staining (400x). FGCs were ingesting a 1030-micron CaHA particle (white arrow), while macrophages were ingesting particles smaller than 10 microns in the dermis. Around FGCs there were coarse and thicker (reinforced) collagen fibers that differed from pre existing, thin, regularly organized patterned collagen fibers. Asteroid bodies (ABs) were visible in FGCs. The right upper giant cell contained a Schaumann body (SB). Many fibroblasts (*) were found around FBCs.

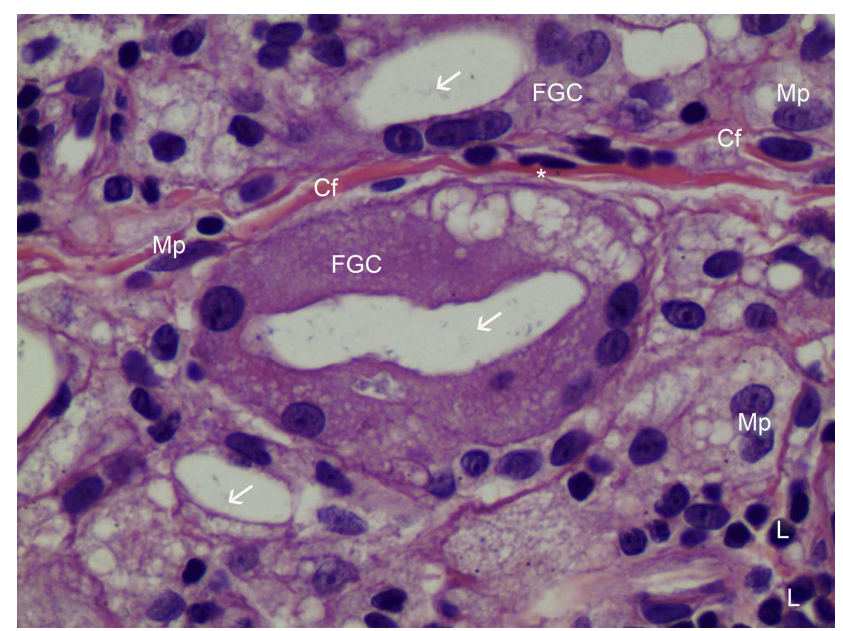

Figure $9 \mathrm{H \& E}$ staining of FGC (400x magnification). Very thin new collagen fibers (Cf) were observed in the dermis between two FGCs and these finding showed collagen fibers were continually created as inflammation processes, if foreign body materials (CaHA particles) existed. Foamy (from 12 o'clock to 3 o'clock) FGCs ingested CaHA particles (white arrows). FGC nuclei were peripherally placed and were overlapping each other. Numerous fibroblasts $(*)$, macrophages $(\mathrm{Mp})$, and lymphocytes (L) were found around FGCs.

micron CaHA-particles" (>40 CaHA-Ps) assumed that particles were injected 1 month ago from the biopsy and "smaller than 20 micron CaHA-particles" ( $<20$ CaHA-Ps) must have been injected 7 months ago from the biopsy. Each stages of wound healing processes and foreign body reactions (near the CaHA particles at 1 month and 7 months after injection on ischial dermis) were found in the same slides. (Figures 5 and 16).

New collagen fibrils and bundles showed different shapes from pre-existing collagen with regular pattern and appearance

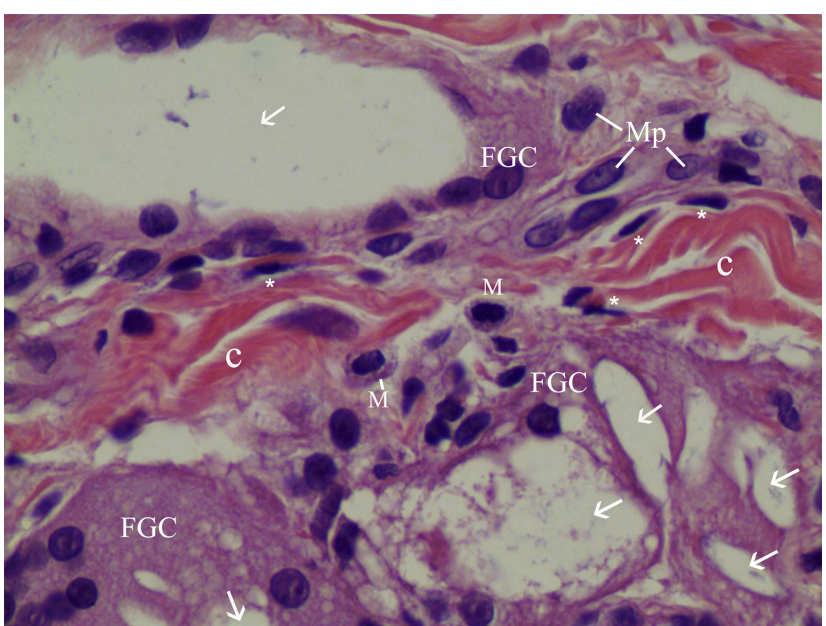

Figure 10 Mast cells and FGCs in $400 \times$ H\&E staining. Two mast cells (M) were observed in the dermis, alongside three FGCs ingesting $\mathrm{CaHA}$ particles. $\mathrm{CaHA}$ particles (white arrow) were visible always in FGCs. Thick, irregularly arranged, fibroblast-produced reinforced collagen fibers (C) were seen between FGCs. Numerous fibroblasts $(*)$ surrounded the matured collagen fibers (C).

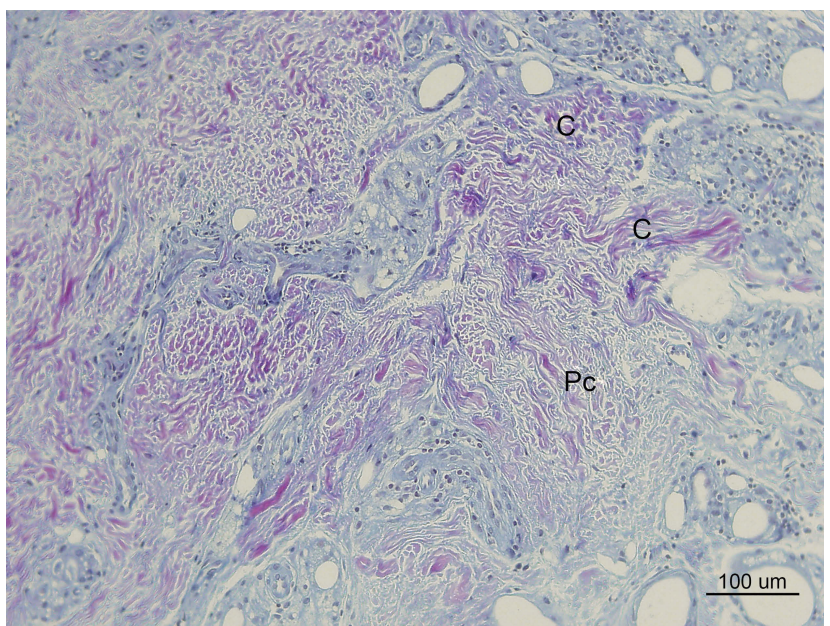

Figure I I Herovici's staining (I00x magnification) in the same dermis area as the H\&E stain in Figure 5. Herovici's stain distinguishes red-stained mature reinforced collagen $(\mathrm{C})$ and red-stained existing collagen $(\mathrm{Pc})$ prior to filler injection from newly formed (young) collagen (blue). Around filler particles and giant cells, abundant new or immature collagen or type-3-Collagen and reticular fibers were observed and stained blue. The reinforced mature collagen (C) showed a thicker and irregular pattern, while pre existing collagen $(\mathrm{Pc})$ showed a regular pattern with regular thickness.

in H\&E, Trichrome, PSR, and IHC stains. Dense, large deposits of thick, irregularly shaped collagen bundles (bigger than 20 microns) were observed around cleaved small-sized CaHA particles ( $<20$ CaHA-Ps) and foreign body giant cells (FGCs). The thicker collagen bundles were assumed to be collagen fibers of 7 months time-frame of stimulation and maturity. Smaller than 3-micron-diameter collagen fibrils were found in all areas of biopsy slide around CaHA-Ps and FGC, and might be the new collagen fibrils recently made by fibroblasts 


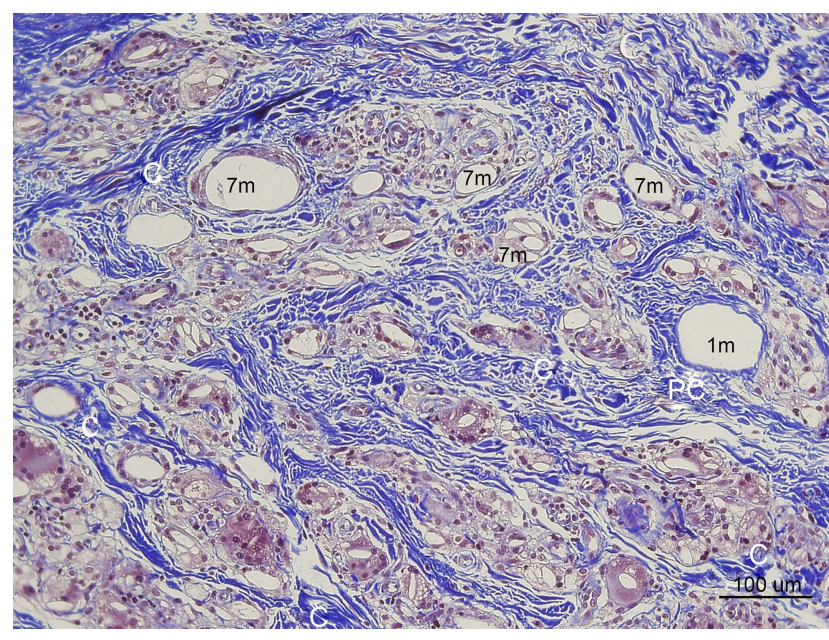

Figure 12 In Masson's Trichrome stain, collagen stained blue (100x magnification). Thick irregularly arranged and blue-stained collagen bundles $(C)$ deposited around CaHA particles $(7 \mathrm{~m})$ injected 7 months pre-biopsy, while finer, thinner, and more indistinct blue collagen fibers were assumed to be pre existingd collagen around 40 micron (recently injected) particles ( $1 \mathrm{~m}$ ). Abundant FGCs (stained light brwon) and its nuclei (dark brwon dots) observed around smaller CaHA particles. (Pre existing collagen fibers: "Pc", Reinforced mature collagen: "C").

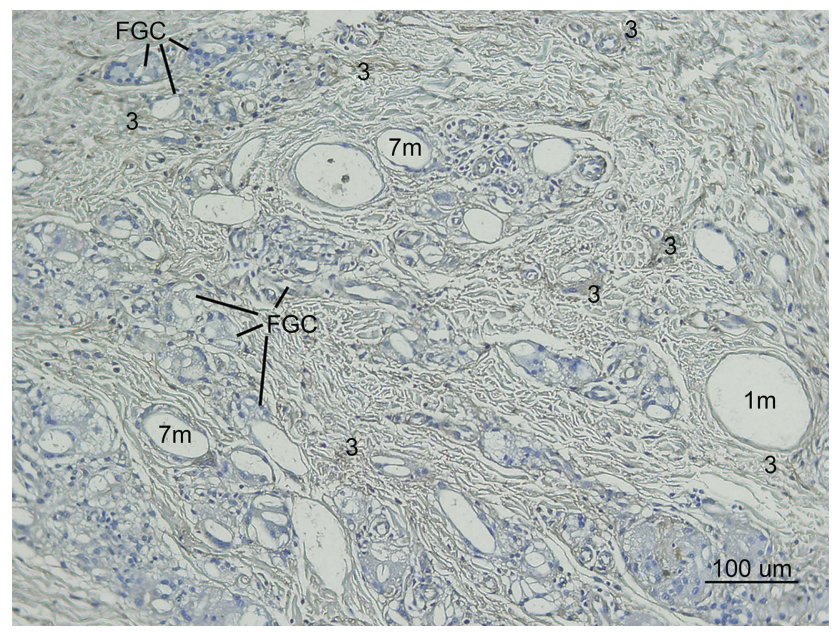

Figure I3 Type III Collagen antibody stain (100x) in the same area of Figure II, 12, I4. Collagen-III fibers (3) appeared brown around and between particles $(1 \mathrm{~m}, 7 \mathrm{~m})$ in the dermis. The shape was in various thicknesses, from thick and densely stained Collagen-III to very thin Collagen-III fibers. Abundant type-III collagen was observed even 7 months after injection of $\mathrm{CaHA}$ filler and this is very first finding. This means new collagen (Type II I and I both) was created continually if CaHA particle existed. Abundant FGCs (stained light blue) and its nuclei (dark blue) observed around smaller CaHA particles in Type-III collagen antibody stain.

through foreign body reactions. During maturation, collagen fibers or collagen bundles may become continuously thicker, if foreign body materials (CaHA-Ps) existed (Figures 5, 12-15).

Fine new collagen fibrils (one layer) were found being in contact with $>40$-CaHA-Ps (denoted as " $1 \mathrm{~m}$ " in Figure 5, 6, 12, 14) without FGCs. These findings showed an early stage of tissue reaction, such as aggregation of fibroblasts mainly with less giant cells around

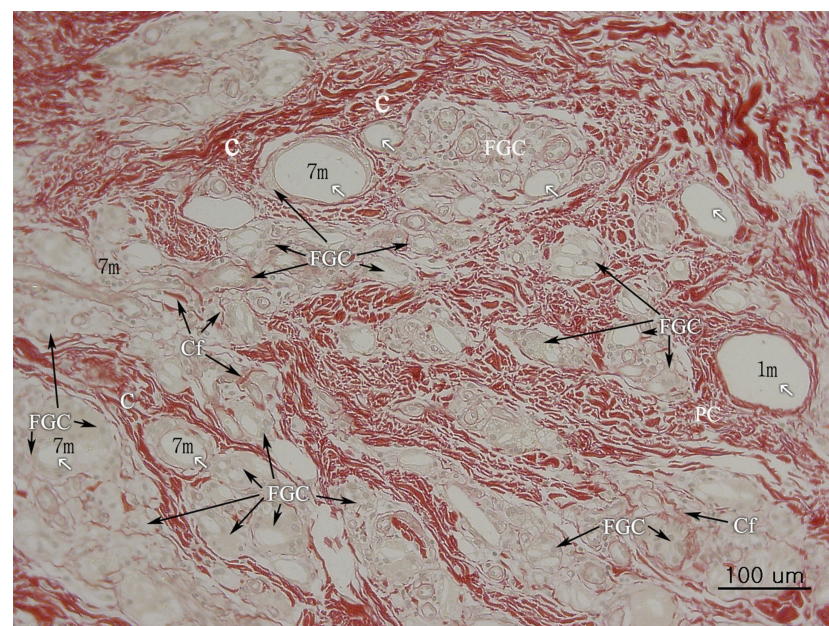

Figure 14 Non polarized PSR staining (100x). Collagen stained red. Pre existing collagen $(\mathrm{Pc})$ showed regular arrangements, whereas thicker irregular patterned matured and reinforced collagen fibers $(C)$ were thicker, denser, and differed in shape and arrangement. A thin, pinkish, and single layer ( $\mathrm{C} f$ ) was visible around a recently injected particle $(\mathrm{I} \mathrm{m})$ and between FGCs, suggesting a recently made collagen fiber. Abundant Very thin and new collagen fibers (Cf) were observed between FGCs.

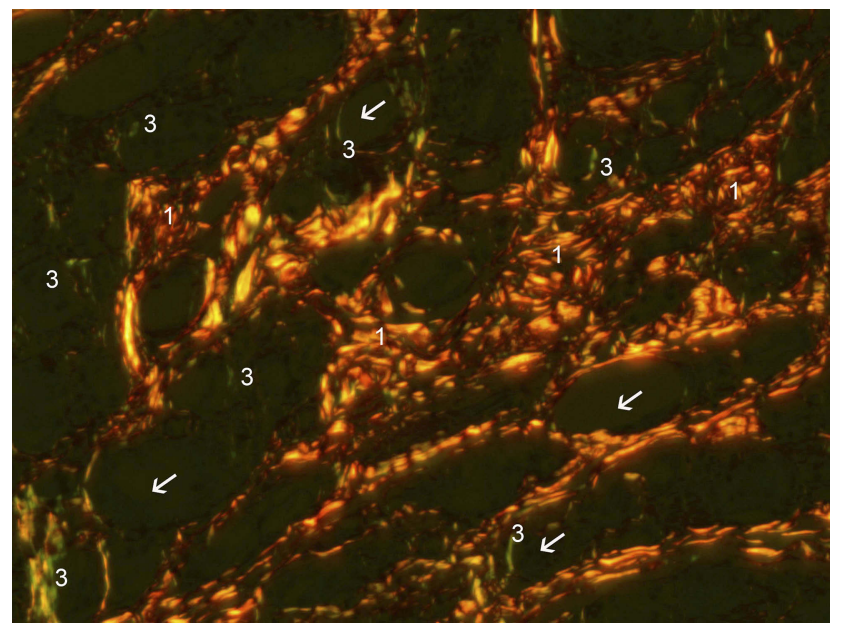

Figure I5 Polarized PSR (200x). Collagen-I stained orange-red (I) and Collagen-III stained green-yellow. Fine green lines (3) of Collagen-III are shown to be in contact with CaHA particles (white arrows).

$>40$-CaHA-ps that must have been injected in the booster session, 1 month before the biopsy (Figure 6). But in other areas of late-stage tissue reaction after the first injection session, all new collagen bundles were subsequently separated from <20-CaHA-Ps by FGC infiltrations (Figure 7). Chronic inflammation was observed around cleaved $<20$-CaHA-Ps (Figure 5, white arrows) with numerous FGC (Figure 7). Less than 20-CaHA-Ps showed irregular shape and were surrounded by numerous FGCs, suggesting that biodegradation had occurred already and must have been injected 7 months before the 


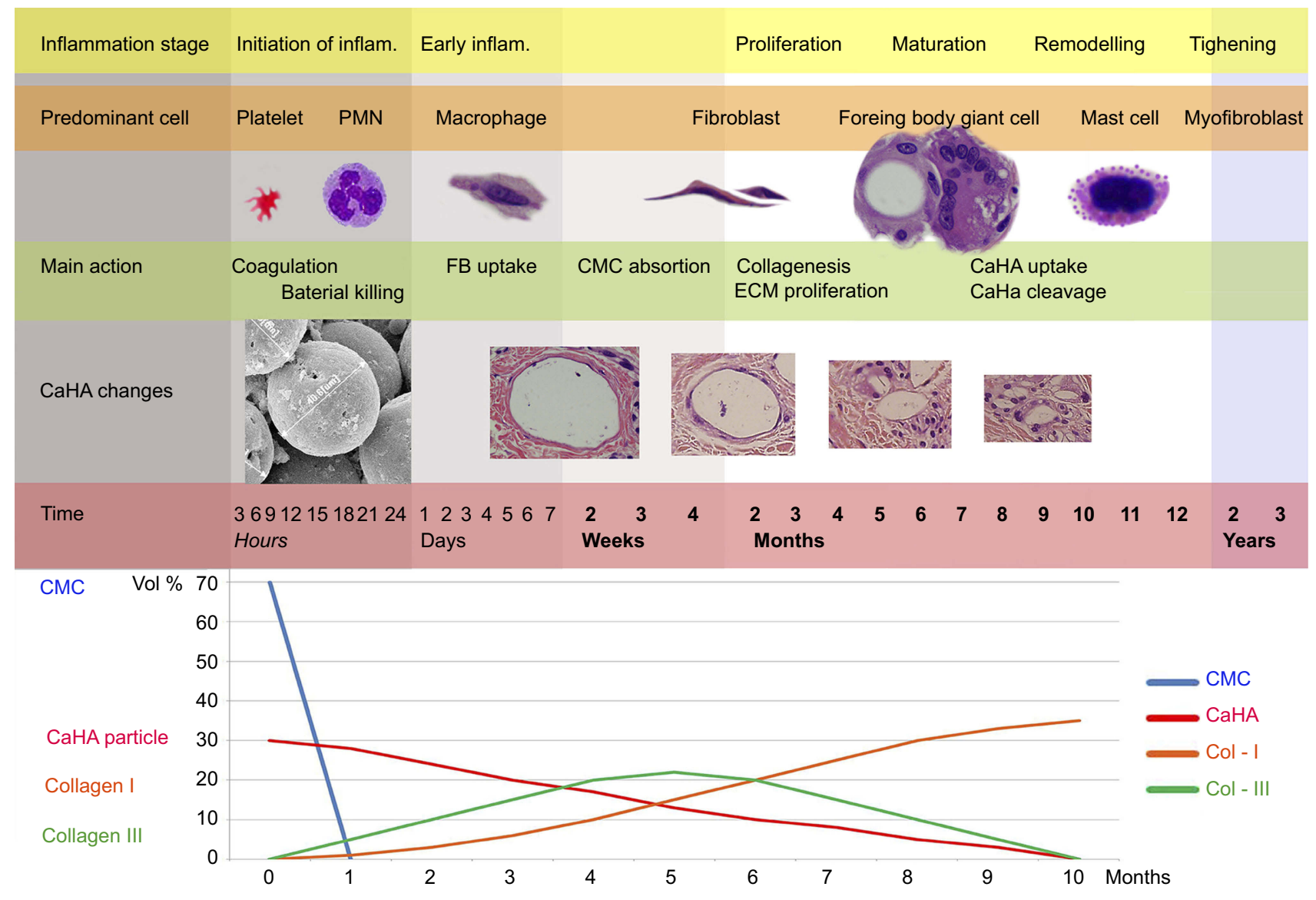

Figure 16 Changes in immune cell appearance, shape, and size of CaHA particles, and collagen levels. (Upper) Immune cell appearance at corresponding stages of inflammation. Within the first few minutes of injury, platelets in the blood begin to stick to the injured site. Platelet-derived growth factors are released into the wound that causes the migration and division of cells during the proliferative phase of the inflammation phase. Within a few hours, polymorphonuclear neutrophils (PMN) arrive at the wound site and become the predominant cells in the wound for the first 2 days after the injury or injection, and phagocytosis debris and kill bacteria. Then, macrophages appear in the wound or injected area for phagocytosis bacteria and damaged tissue. Macrophage also functions in regeneration for wound healing and also stimulates to create granulation tissue and lay down a new extracellular matrix. When macrophages cannot clean the foreign body, macrophages fused together and became a foreign body giant cell (roughly I month after injection of CaHA filler). In fibroplasia and granulation tissue formation, fibroblasts grow and form a new, provisional extracellular matrix by excreting collagen and fibronectin. The re-epithelialization process does not occur after filler injection. (Middle) Changes in the shape and size of CaHA particles. Before injection, the size of CaHA particles was 36-52 microns. As time passes, the size was reduced, and particles were cleavaged. Largersized CaHA particles were surrounded only by FGCs and less than 10-micron CaHA particles by macrophages and FGCs both. (Bottom) Changes in collagen levels were associated with the amount of CaHA particle and immune cells. Initial collagen created with stimulation may be type III. As time passes, the amount of Collagen-III decreased and was replaced by Collagen-I in end stages of inflammation after CaHA biodegradation. This Collagen-I deposition may maintain somewhat the volume as CMC portion absorption. CMC was absorbed about I month after injection. CaHA particles were absorbed about I year after injection.

biopsy. Less than 10-CaHA-Ps were then engulfed and degraded by macrophages. Less than 20-CaHA-Ps were probably injected 7 months pre-biopsy, as the area demonstrated late inflammation (Figures 7-10), numerous FGCs, and macrophages around the biodegraded particles (Figure 16).

Mast cells, which are key players in inflammation, were found in low numbers around $<20$-CaHA-Ps, along with CaHA-stimulated fibroblasts that produced collagen at late wound healing stages (Figure 10). Plasma cells were not found in all specimens suggesting a lower degree of foreign body reactions without problematic granuloma. Three eosinophils were found in 50 slides (Figure 7), and the direct correlation between the presence of eosinophils and tissue reaction by $\mathrm{CaHA}$ filler was in question but it may have strong reactions. Numerous monocytes including lymphocytes were observed around cleaved $<20$ CaHA-Ps and FGCs (Figures 8-10).

Asteroid bodies (ABs) were observed in FGCs as stellate inclusions projecting from a central core of the FGC cytoplasm. ABs were 10-15 microns in size and had 7-18 star-like projections and vacuolated surrounding cytoplasm (Figure 8). Schaumann bodies (SBs) were found in FGCs and had ingested a CaHA-P (Figure 8).

In Herovici's stain, new collagen fibrils and bundles were stained blue. Distribution of new collagen fibers in Herovici's 
stain was different from type III collagen (Collagen-III) IHC stain and polarized PSR stain. Mature (old) Collagen-I fibers were stained red, while newly formed (young) collagen fibers and Collagen-III stained blue in Herovici's stain. ${ }^{14}$ Collagen bundles around filler particles were stained blue, indicating immaturity of collagen that including Collagen-III, and their abundance of the blue stained areas suggested that substantial CaHA-induced neocollagenesis were whether the fibers of type I or III collagen (Figure 11).

In Trichome stain all, new and pre existed, collagen fibrils and bundles were stained blue. Among them the pre-existing collagen fibers (denoted by "PC", Figure 12) showed regular pattern and size compared to the rough-patterned thick collagen fibers (denoted by " $\mathrm{C}$ " in Figure 12) found around FGCs. These coarse collagen pattern had mild similarities to scar tissue, therefore assuming to be the reinforced collagen with stimulation. Additionally, very fine thin collagen fibrils were found around and between CaHA particles. These new thin collagen fibrils and thicker, reinforced coarse collagen bundles might have tightened the skin and improved skin folds (Figure 12).

In Collagen-III-IHC stain, Collagen-III fibers were also found, stained brown, near all CaHA-Ps including $<20$-CaHA-Ps and >40-CaHA-Ps (Figure 13), suggesting that Collagen-III were produced near the CaHA-Ps continuously. New collagen-III fibers were synthesized primarily near the CaHA repeatedly when $\mathrm{CaHA}$ particles existed, and in this sequence new collagen-I fibers synthesized around CaHA-Ps repeatedly. This repeated neocollagenesis occurred like an "onion pattern".

Non polarized PSR staining highlighted the differences in collagen shape and color. Very thin new collagen fibers were stained red and a "cloudy-red thin (pink)" layer of new collagen fibril around >40-CaHA-Ps (Figure 12 " $1 \mathrm{~m}$ ") were likewise stained red. Thick, denser-red and coarser collagen fibers ("C") found around FGCs and <20-CaHA-Ps may have been reinforced by stimulation caused by inflammation from the previous 7 months (Figure 14). On the other hand, pre existing collagen ("PC") maintained its specific pattern and thickness and was similarly shaped, unlike the dense thicker irregularly shaped revealed from a pattern of collagen fibers in hypertrophic scar. CaHA ultimately has significantly increased type I collagen (Collagen-I) formation during the 7 months (Figure 14).

In polarized PSR (200× magnification), Collagen-I were stained orange-red while Collagen-III were stained green-yellow. There were thin green lines around all CaHA particles (Figure 15). These Collagen-III fibers were synthesized continuously around CaHA-Ps $<20$ CaHA-Ps or $>40$-CaHA-Ps and simultaneously CollagenI synthesized in an onion-shaped pattern continuously around CaHA-Ps until CaHA absorption. In polarized PSR, the amount of Collagen-III was much lesser than in Herovici' stain slide (Figure 11), which means new collagen in Herovici' stain was not only Collagen-III. In previous articles, Herovici's stains primarily represented in Collagen-III as blue; however, this study showed for the first time that new collagen fibers stained blue in Herovici' stain are not only Collagen-III (in polarized PRS stain) but also suggest other types of collagens.

Carboxymethylcellulose (CMC) portion of the filler material was absent and not found in most specimens 1 month after injection. An additional new finding suggests that $\mathrm{CMC}$ is absorbed by macrophages or FGCs within 1 month after the filler injection. In the author's other biopsy study, CMC gels were found and were up-taking by macrophages.

Small capillaries, including the RBC (empty black arrows, Figure 7), were discovered witthin thin endothelial cell layers, which reflected their newly made composition. Endothelial cells were thin and small, indicating their newness and neovascularization.

In most giant cells, the nuclei were centrally placed and overlapped each other showing typical shape of FGCs (Figure 7; black arrow), and among them, the ingested CaHA particles might have displaced the nuclei of FGCs peripherally. Interestingly in a few of the giant cells, the nuclei were arranged along the border like a horseshoe shape showing a typical pattern of Langhans giant cell (Figure 7; red arrow).

\section{SEM finding}

CaHA-Ps were roughly 40 microns (37-52 microns) in size, with smooth and porous (1-3 microns) surfaces and fully rounded shapes (Figure 17).

\section{Discussion}

According to Johns Hopkins Medicine, a sore can develop if blood supply is cut off for more than 2-3 hrs. Pressure ulcers are usually caused by (1) Continuous pressure: if there is pressure on the skin on one side with bone on the other, the skin and underlying tissue may not receive an adequate blood supply. (2) Friction: for some patients, particularly those with thin, frail skin and poor circulation, movements like turning and moving may damage the skin, which increases the risk of bedsores. (3) Shear: if the skin moves one way while the underlying bone moves in 


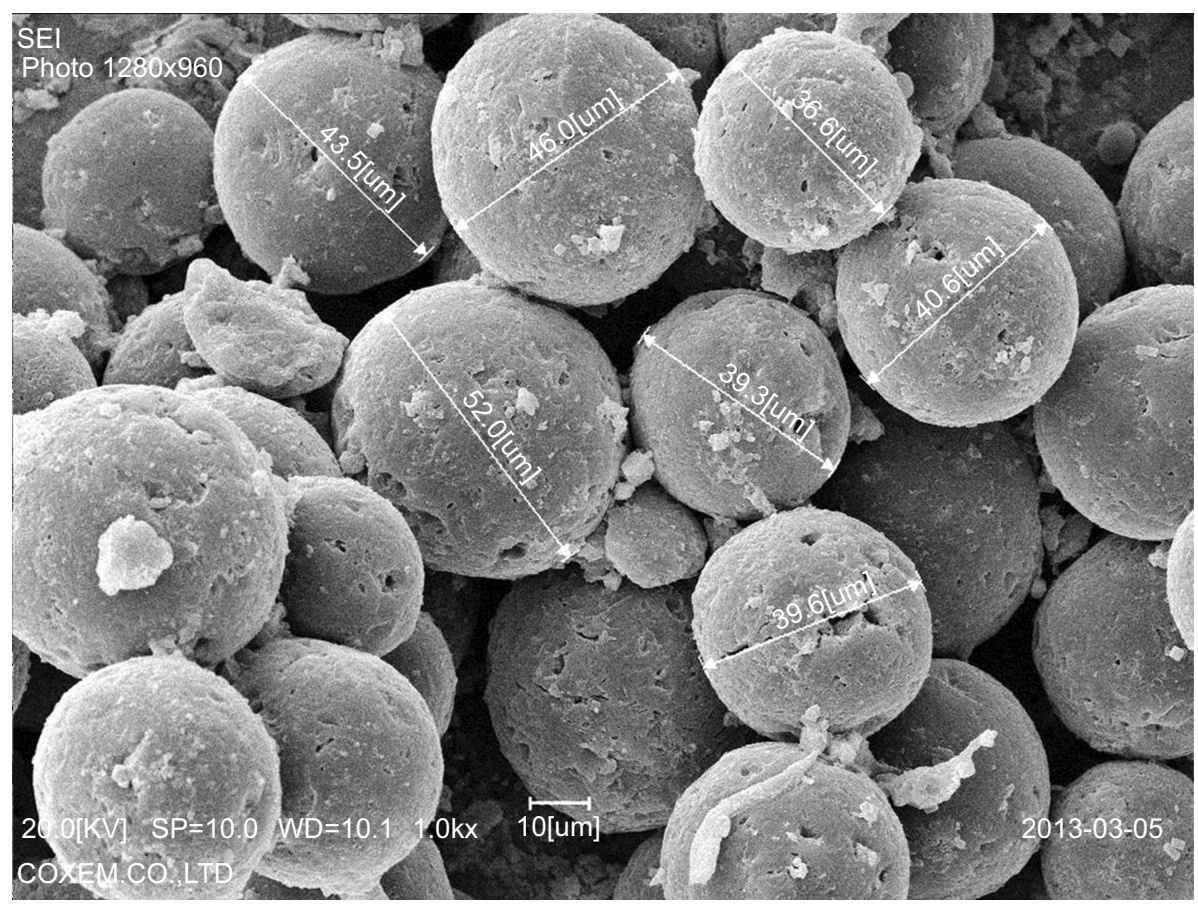

Figure 17 SEM demonstrating CaHA filler particle size as 36-52 microns at 1000x magnification. Particles had smooth surfaces, were completely spherical, and had about 3micron-sized small holes in the surface.

opposite directions, it creates a risk of shearing where cell walls and minute blood vessels may stretch and tear.

Factors that increase pressure ulcer risks include the following: (1) older age as skin becomes thinner and more vulnerable; (2) reduced pain perception due to nerve-affecting injuries like the spinal cord as they may not notice the sore; (3) poor blood circulation due to diabetes, vascular diseases, smoking, and compression; (4) poor diet, from a lack of protein, vitamin $\mathrm{C}$, and zinc; (5) reduced mental awareness due to disease, injury, or medication, which can reduce the patient's ability to take preventive action; (6) incontinence of urine or feces can cause areas of permanently moist skin, which increases the risk of skin breakdown and damage; and (7) either a low or high body mass index.

A previous phase of chronic sitting pressure sore focuses a localized injury to the skin and its underlying soft tissue usually over ischial prominences, as a result from long-term pressure during the chronic sitting position. After a few hours of sitting position, the ischial skin can change to purple or maroon localized discolored skin from the decrease in blood circulation of the underlying soft tissue. The area may be preceded by tissue that is painful, firm, mushy, or boggy as compared to its adjacent tissue for the loner sitting position. In Stage I of pressure sore, intact skin has non-blanchable redness over a localized area commonly with a bony prominence, but this usual sitting position does not entice real pressure sore in a healthy person. The previous phase of sitting pressure sore with soft tissue atrophy and pigmentation can occur after continued repeated pressure and decreased circulation. This article indicatted a previous phase of the chronic sitting pressure sore. There is no relationship between mechanotransduction and pressure sores because they reflect different bodily reactions to trauma. Callus forms after exercise with weight-bearing stress from strong grips such as golf and tennis. However, the pressure sore is created by decrease of blood circulation, resulting in fibroblasts and no healthy collagen production.

This study demonstrated that initially the macrophage cannot engulf $>40$-CaHA-Ps and aggregate to FGCs cells, and then FGCs engulfed $>40$-CaHA-Ps at later stage of inflammation, around 1 month after injection, then macrophages also ingested biodegraded small-sized and destroyed particles less than roughly 10 microns in size at the much later stages after FGCs destroyed CaHA-Ps at end stage of tissue reaction after CaHA filler injection. The author's study proved that after injections of Ca-HA filler into the human dermis or subdermis, FGCs normally were found in all cases without any infection signs or granuloma symptoms. Therefore, the presence of FGSs does not mean granuloma because granulomas showed more aggressive 
tissue reactions and different patterns of FGCs with chronic specific clinical symptoms. FGCs always disappeared after CaHA biodegradation, whether true granuloma or usual cases after CaHA injection. Thus, if even true granuloma occurred, it can be controlled easily after biodegradation of CaHA-P for an estimated 1 year post injection.

Initial tissue irritation and initial swelling within the first few weeks may partially be caused by the CMC component because CMC contains glycerin. This irritation from $\mathrm{CMC}$ may affect the pseudo-volumization result with the initial a few days. The real volume effect by CMC disappears slowly in 1 month. Physicians must notify their patients that volume loss from CMC absorption would occur 1 month after all procedures using CaHA-filler. However, the CaHA component remained until 10 months and stimulated new collagen, partially replacing the volume initially lost following both CMC absorption and bio-resorption of CaHA component.

New tissues around FGCs and CaHA-Ps were observed and may be composed of fibroblast-produced new collagens, glycosaminoglycans, reticular and elastic fibers, and glycoproteins. New collagen fibers were confirmed in all biopsy specimens, and new reticular fibers were confirmed in polarized PSR, Collagen-III IHC, and Herovici's stain. Previous articles described particle size after injection into the soft tissue. Particles smaller than 15-20 microns are phagocytosed easily; particles over 50 microns may cause excessive inflammation and granulomas. ${ }^{15}$ The average particle size detected by SEM in the study was an estimated 40 microns (37-52 microns), where 40 microns was optimal for injection, avoiding macrophage uptake and excessive tissue reaction. Uptake of larger CaHA particles occurred 1 month after injection by FGCs, which cleaved and divided the particles into sizes less than 10 microns allowed subsequent uptake by macrophages in the end stages.

Phagocytosis by macrophages had not yet occurred around >40-CaHA-Ps immediately after injection; thus, macrophages were absent around the particle in the initial stage. This was probably because the particle (about 40 microns) was too large to be engulfed by macrophages and because of its CaHA component, pre-existing naturally within the human body. This inherent quality may have prevented immune cell recognition within 1 month or at 1 month ("early stage") which explains their lack of cell attachment. Individual macrophages were less seen ingesting $>40$-CaHA-Ps in the early stage; however, at the "later stages" (7 months after injection), abundant amounts of FCGs and macrophase appeared after the injection due to many macrophage fusions to FCGs. ${ }^{14}$ The surface change from CaHA-Ps' initial smoothness to roughness suggested their recognition as large foreign body reactions with greather antigenicity. ABs found in FGCs had vacuolated cytoplasm containing lamellated, lipoidal, myelinated figures probably associated with SB formation. ${ }^{16}$ ABs can also be found in FGCs in granulomas where they can contain cytoplasmic threads $^{15}$ or microtubular constituents ${ }^{17,18}$ (Figure 8).

After 1 year, CaHA is going to be completely metabolized, while giant cells and other immune cells will also be no longer present. Additionally after 1 year, even in cases of infection from contamination during injection or later, true granulomas alos is going to be disappeared. While granulomas always contain FGCs, but only the presence of FGC does not always indicate granuloma formation because granulomas must be also associated with clinical symptoms including swelling, redness, and pain. FGCs result from macrophage fusion in response to the presence of a large foreign body ${ }^{19}$ and always exists with CaHA implants that produce chronic inflammation and foreign body reactions at injection sites. ${ }^{20}$ Compared to polycaprolactone (PCL)-based fillers lasting 3-4 years, granulomas after PCL injection from contamination will last greater than 4 years. In a previous biopsy study by the author, a PCL filler (Ellansé M, Sinclair Pharma, UK) had lasted over 4 years which increased the risk of granulomas. ${ }^{21}$

Following implantation, CaHA filler metabolized to calcium and phosphate ions, which are normally present in the body ${ }^{22}$ and resorbed after a year, at the same time the space was gradually replaced by infiltrating fibrovascular stroma maintaining overall volume despite CaHA volume decrease. Yet within thte first month, $\mathrm{CMC}$ absorption does not maintain overall volume because new collagen formation has not yet started until after roughly 1 month post-injection. After CaHA bioresorption, FGCs, macrophages, and lymphocytes are going to be disappeared because there was not antigenic material nor foreig body. In Herovici, Trichrome, PSR, and IHC staining after CaHA implantation, Collagen-III has produced around all CaHA particles (like the larger 1-month and 7-month particles) but then decreased following CaHA biodegradation and the space by Collagen-III may be replaced by Collagen-I. Compared to previous studies, Collagen III repeatedly produced near the CaHA particles in the onion-shaped pattern over a lasting period of time. Simultaneously, mainly Collagen-I production was stimulated. In previous studies that use only PRS stain to confirm Collagen III, only small amounts of Collagen-III ws found at 6 month after $\mathrm{CaHA}$ injection and gradual 
replacement by collagen Type $\mathrm{I}$ in the process of remodeling. ${ }^{23}$ But in this study, Collagen-III was found all CaHA-Ps in 7-month biopsies in Herovici's stain, and the presence of CaHA-Ps stimulated the synthesis of Collagen-III continuously (Figure 11 and 16).

Mast cells may participate in angiogenesis and neocollagenesis, ${ }^{24}$ whereas significantly in this plasma cells were not found in all biopsies. Mast cells were observed around CaHA particles for the first time in this study (Figure 10). In the author's previous studies, eosinophils and plasma cells detected in granuloma cases appeared 1 year after injection of Ellansé-M. ${ }^{21}$ The presence of eosinophils and plasma cells indicates excessively increased inflammation.

In normal wound healing, fibrin clots release chemotactic factors which starts the initial inflammatory phase. Neutrophil is the first cell to infiltrate and then replaced by macrophages a few days later during the beginning of the late-inflammatory phase. Finding the neutrophils during this initial phase at 1 month postinjection proved difficultbetween the late inflammation phase and proliferation phase. Some of the wound healing processes following CaHA injection included early inflammations with macrophages and fibroplasia. In the proliferation phase, granulation tissues formed around the CaHA injection site and consisted of new blood vessels (Figure 7), fibroblasts, inflammatory cells, endothelial cells, myofibroblasts, and provisional ECM components. CaHA may therefore have stimulated fibroblast to produce collagen, fibronectin, glycosaminoglycans, elastin, glycoproteins, and proteoglycans, ${ }^{25}$ to construct a hydrated ECM and to enable cell migration and subsequent maturation into a non-injured tissue ECM. In the author's study, granulation tissue was observed around 1 month post injection, and At 7 months postinjection, there were more abundant granulation tissues stained blue in Herovici's stain (Figure 11). Yet in this study, distinct fibrous encapsulation was not found (Figure 11), because either the CaHA particle was too small or CaHA content is same material as our body' composition.

Within foreign body reaction, macrophages and giant cells play great roles interacting and cross-talking with each other. Foreign body reaction occurs in a series of processes. The first process is protein adsorption on the foreign body surface following with macrophages adhesion. The second process with macrophages is alternatively activated by IL-4 and IL-13, which are cytokines produced in T helper (TH)2-type responses, hence the term M2 macrophages. Macrophages migrate to local sites of injury and infection where they contribute to acute and chronic these types of inflammations. In addition, they can initiate tissue remodeling and resolve inflammation. There are two different ways of differentiating and activating monocytes into macrophages that depend on specific growth factors, receptors, and cytokines. Mirroring the TH1-type and TH2-type nomenclature, the classical activation provides M1 cells and the alternative activation provides M2 cells. Macrophages express both of these receptor types. However, it was not possible to distinguish between $\mathrm{Ma}$ and M2 cells during this study.

Recovery from needle or cannula injuries may cause minimal inflammations and proliferation phases, not only with fewer inflammatory factors secreted and degradation of remaining ones, but also with fewer neutrophils and macrophages at the wound, therefore may initiating its subsequent stages. ${ }^{26,27}$ Fibroblasts secrete growth factors, stimulate proliferation and inflammation cell migrations to wounds, and mediate ECM and collagen production. ${ }^{28}$ Collagen deposition increases wound strength ${ }^{29}$ while cells contributing to inflammation, angiogenesis, and connective tissue development adhere to and proliferate on fibroblast-generated collagen matrix. ${ }^{30}$ In normal wound healing remodeling starts 1 year post injection of filler injection as collagen levels stabilize and tissue repair initiates. ${ }^{29}$ Collagen-III and fibronectin may be the predominating tensile substances until late maturation when they are replaced by the stronger Collagen-I, ${ }^{31}$ before this, Collagen-III is cross-linked and rearranged along tensile lines. The onset of maturation may vary depending on the amount of CaHA injected and the areas injected, but can last over a year, ${ }^{32}$ during which collagen is continually produced. In addition to being an integral structural component of many organs, collagen-III is also an important regulator of the diameter of type I and II collagen fibrils. Collagen-III is also known to facilitate platelet aggregation through its binding affinity to platelets and therefore plays an important role in blood clotting. Type III collagen is found as a major structural component in hollow organs such as large blood vessels, uterus, and bowel. It is also found in many other tissues together with type I collagen. ${ }^{33-35}$

In giant cells, $\mathrm{ABs}$ and $\mathrm{SBs}$ were found (Figure 8). ABs are found in non-necrotizing granulomas, not in necrotic areas, and no central necrosis was observed. ${ }^{35} \mathrm{SBs}$ had ingested a CaHA-P (Figure 8, SB). In pathology, SBs are calcium and protein inclusions inside of FGCs as part of a granuloma. Many conditions can cause SBs, including sarcoidosis, hypersensitivity pneumonitis, and Berylliosis, uncommonly, Crohn's disease and tuberculosis. ${ }^{36}$ However, the presence of $\mathrm{ABs}$ and $\mathrm{SBs}$ does not always mean 
granuloma, and all FGCs, Abs, and SBs is going to disappear after biodegradation of CaHA filler.

\section{Conclusion}

Depressed soft tissue and skin folds on ischial areas were significantly improved by volumization of subdermal filler injection. The skin quality, roughness, and pigmentation on ischial areas were improved, and these improvements may be caused by intradermal micro-droplet injections of CaHA-filler and may be due to neocollagenesis by numerous fibroblasts and increased micro-blood circulation (neovascularization). Newly made, small capillaries composed with very thin endothelial cell wall were found, and there were one or two erythrocytes within the capillaries. Endothelial cells were thin and small, indicating their newness and neovascularization.

This is the first article to show the scientific evidence of neocollagenesis and tissue reactions after injection of CaHA filler in dermis using various histological stainings, and to show various stages of inflammation, wound healing, and foreign body reaction around CaHA particles. Although numerous fibroblasts were present around CaHA-Ps, plasma cells were absent with very few eosinophils, indicating that excessive inflammation after $\mathrm{CaHA}$ injection had not occurred in contrast with PCL or PLLA filler.

The multi-layered (targeting both intradermal and subdermal layers) injection approach was safe and effectively treated ischial soft tissue atrophy without significant side effects such as infection or delayed swelling or lumps. After a significant period (6 to 12 months) of time, these multilayered injections of diluted $\mathrm{CaHA}$ tightened and remodeled atrophic ischial skin.

New collagen-III fibers were synthesized mainly near the CaHA repeatedly if CaHA-Ps existed, and in sequence, new collagen-I fibers were also synthesized around CaHA-Ps repeatedly like an onion pattern during a significant period (6 to 12 months).

CMC portion (which compose $70 \%$ of the volume of filler) of the filler was not found in this study, and this finding suggests that $\mathrm{CMC}$ is absorbed by macrophages or FGC within 1 month after the filler injection. With the absorption of CMC portion, volumizing effect by filler also may decrease in some degree (about 50\%) within 1 month after injection. Among the decreasing volume by absorption of CMC portion, a partial portion of volume may be filled by neocollagenesis but new collagen or new soft tissue could not replace the total volume of CMC portion. While with the absorption of CaHA portion, the entire volume is going to be replaced by new collagen tissues.

\section{Acknowledgment}

The author would like to thank Ms. Cho Mong-Ee for supporting all.

\section{Disclosure}

The author has no financial interest in any of the products, devices, or drugs mentioned in this manuscript. This is the author's own study for medical science. The author reports no conflicts of interest in this work.

\section{References}

1. Marmur ES, Phelps R, Goldberg DJ. Clinical, histologic and electron microscopic findings after injection of a calcium hydroxylapatite filler. J Cosmet Laser Ther. 2004;6:223-226. doi:10.1080/14764 1704100003048

2. Yutskovskaya Y, Kogan E, Leshunov E. A randomized, split-face, histomorphologic study comparing a volumetric calcium hydroxylapatite and a hyaluronic acid-based dermal filler. J Drugs Dermatol. 2014;13(9):1047-1052.

3. Department of health \& human services. 2015. Available from: http:// www.accessdata.fda.gov/cdrh_docs/pdf5/p050052s049a.pdf. Accessed April 28, 2015.

4. Kim JS. Detailed sonographic anatomy of dorsal hand augmentation with hyaluronic acid and calcium hydroxyapatite fillers. Aesthet Surg J. 2018 Sep 5. doi:10.1093/asj/sjy227. [Epub ahead of print]

5. Fisher GJ, Varani J, Voorhees JJ. Looking older: fibroblast collapse and therapeutic implications. Arch Dermatol. 2008;144:666-672. doi:10.1001/archderm.144.5.666

6. Ashmole I, Bradding P. Ion channels regulating mast cell biology. Clin Exp Allergy. 2013:43(5):491-502. PMID 23600539. doi:10.1111/ cea. 12043

7. Kim JS. Effects of injection depth and volume of stabilized hyaluronic acid in human dermis on skin texture, hydration, and thickness. Arch Aesthetic Plast Surg. 2014;20(2):97-103. doi:10.14730/aaps.2014.20.2.97

8. Egawa M, Oguri M, Kuwahara T, Takahashi M. Effects of exposure of human skin to a dry environment. Skin Res Technol. 2002;8 (4):212-218.

9. Setaro M, Sparavigna A. Irregularity skin index (ISI): a tool to evaluate skin surface texture. Skin Res Technol. 2001;7(3):159163.

10. Del Carmen LP, Da Cunha MM, Zapata AJ, Cherit JD, Gallegos ER. Implementation and analysis of relief pattern of the surface of benign and malignamt lesions of the skin by microtopography. Phys Med Biol. 2005;50(23):5535-5543. doi:10.1088/0031-9155/50/23/008

11. Brown CA, Charles PD, Johnsen WA, Chesters S. Fractal analysis of topographic data by the patchwork method. Wear. 1993;161:61-67. doi:10.1016/0043-1648(93)90453-S

12. Brown CA, Johnsen WA, Charles PD Method of quantifying the topographic structure of a surface. US Patent No. 5307292, 1994. doi:10.3168/jds.S0022-0302(94)77044-2

13. Konstantin A, Christopher AB, Klaus PW. Scale-sensitive fractal analysis using the patchwork method for the assessment of skin roughness. Skin Res Technol. 2001;7:164-167. doi:10.1034/j.16000846.2001.70304.x 
14. Freitas RA Jr. Alumina and sapphire particles. NanomedicineBookSite. Available from: Available from: http:/www.nanomedicine.com/ NMIIA/15.3.5.5.htm. Accessed December 13, 2008

15. Morhenn VB, Lemperle G, Gallo RL. Phagocytosis of different particulate dermal filler substances by human macrophages and skin cells. Dermatol Surg. 2002;28:484-490.

16. Kirkpatrick C, Curry A, Bisset D. Light- and electron-microscopic studies on multinucleated giant cells in sarcoid granuloma: new aspects of asteroid and Schaumann bodies. Ultrastructural Pathol. 1988;12:581-597. doi:10.3109/01913128809056483

17. Cain H, Kraus B. Asteroid bodies: derivatives of the cytosphere. An electron microscopic contribution to the pathology of the cytocentre. Virchows Arch B Cell Path. 1977;26:119-132.

18. Gadde P, Moscovic E. Asteroid bodies: products of unusual microtubule dynamics in monocyte derived giant cells. An immunohistochemical study. Histol Histopathol. 1994;9:633-642.

19. UWEB: Research: Biomaterials Tutorial. Available from: www. uweb.engr.washington.edu. Accessed 2010

20. Foreign Body Giant Cell at the US National Library of Medicine Medical Subject Headings (MeSH). Available from: https://meshb. nlm.nih.gov/record/ui?ui=D015743. Accessed June 2011.

21. Kim JS. Changes in dermal thickness in biopsy study of histologic findings after a single injection of polycaprolactone-based filler into the dermis. Aesthet Surg J. 2019; Epub ahead of print. doi:10.1093/asj/ sjz050

22. LeGeros RZ. Biodegradation and bioresorption of calcium phosphate ceramics. Clin Mater. 1993;14:65-88. doi:10.1016/0267-6605(93) 90049-D

23. Berlin AL, Hussain M, Goldberg DJ. Calcium hydroxylapatite filler for facial rejuvenation: a histologic and immunohistochemical analysis. Dermatol Surg. 2008;34(Suppl 1):S64-S67. doi:10.1111/j.15244725.2008.34245.x

24. Norrby K. Mast cells and angiogenesis. APMIS. 2002;110(5):355-371.

25. Romo T, Pearson JM 2005. Wound healing, skin. Available from: https://commons.wikimedia.org/wiki/File:Wound_healing_phases. svg. Accessed September
26. Torre J, Sholar A. Wound healing: chronic wounds; 2006). Available from http://emedicine.medscape.com/article/1298452overview. Accessed October, 2008.

27. Newton PM, Watson JA, Wolowacz RG, Wood EJ. Macrophages restrain contraction of an in vitro wound healing model. Inflammation. 2004:346 28(4):207-214. PMID15673162. doi:10.1023/B:IFLA.00000 49045.41784.59

28. Kuwahara RT, Rasberry R. Chemical peels, 2007. 15:2007-2009. Available from: Emedicine.com. Accessed September

29. Greenhalgh DG. The role of apoptosis in wound healing. Int $J$ Biochem Cell Biol. 1998:30(9):1019-1030. PMID 9785465. doi:10.1016/S1357-2725(98)00058-2

30. Ruszczak Z. Effect of collagen matrices on dermal wound healing. Adv Drug Deliv Rev. 2003:55(12):1595-1611. PMID 14623403. doi:10.1016/j.addr.2003.08.003

31. Dealey C. The Care of Wounds: A Guide for Nurses. Electronic book. Oxford: Malden, Mass. Blackwell Science; 1999.

32. Mercandetti M, Cohen AJ. Wound healing: healing and repair; 2005. Available from: Emedicine.com. Accessed May, 2017.

33. Liu X, Wu H, Byrne M, Krane S, Jaenisch R. Collagen-III Type III collagen is crucial for collagen I fibrillogenesis and for normal cardiovascular development. Proc Natl Acad Sci USA. 1997;94 (5):1852-1856. doi:10.1073/pnas.94.5.1852

34. D'hondt S, Guillemyn B, Syx D, et al. Type III collagen affects dermal and vascular collagen fibrillogenesis and tissue integrity in a mutant Col3al transgenic mouse model. Matrix Biol. 2018;70:72-83. Epub 2018 Mar 15. doi:10.1016/j.matbio.2 018.03 .008

35. NJ T, Pezzone MA, Brown BN, Badylak SF. Quantitative multispectral imaging of Herovici's polychrome for the assessment of collagen content and tissue remodelling. J Tissue Eng Regen Med. 2013;7(2):139-148. doi:10.1002/term.508

36. Cain H, Kraus B. Asteroid bodies: derivatives of the cytosphere. An electron microscopic contribution to the pathology of the cytocentre. Virchows Arch B Cell Pathol. 1977;26(2):119-132.

\section{Publish your work in this journal}

Clinical, Cosmetic and Investigational Dermatology is an international, peer-reviewed, open access, online journal that focuses on the latest clinical and experimental research in all aspects of skin disease and cosmetic interventions. This journal is indexed on CAS.
The manuscript management system is completely online and includes a very quick and fair peer-review system, which is all easy to use. Visit http://www.dovepress.com/testimonials.php to read real quotes from published authors. 